\title{
A Search for the 1.36-cm Water-Vapor Line in Venus
}

\author{
F. D. Drake \\ Center for Radiophysics and Space Research, Cornell University, Ithaca, N.Y.
}

A search for the water-vapor line was made by Stevens, Drake, Jones, Sato, Stelzried, Potter, and others at the Jet Propulsion Laboratory at the California Institute of Technology. A $30-\mathrm{ft}$ diam altazimuth radio telescope at the Goldstone Tracking Station was used. The radiometer was a conventional superheterodyne radiometer with a backward-wave oscillator as the local oscillator, which permitted frequency scanning from 20.5 to $24.0 \mathrm{Gc} / \mathrm{s}$. On-off observations were made of Venus throughout the day, with frequent changes of the frequency observed. Extinction was measured using the Moon as a radio source. It was found, however, that extremely accurate extinction values for all frequencies could be derived from the theory of Barrett and Chung, using the radiosonde measurements made at the Edwards Air Force Base some 50 miles from the observing site. Measurements were made of the antenna gain, using a remote transmitter at a relatively high elevation on Tiefort Mountain. Although these measurements were entirely self-consistent, they differed markedly from theoretical predictions of the antenna gain as a function of frequency, thus producing some uncertainty as to the true value of antenna gain. Measurements were made at eight discrete frequencies between 20.5 and $24.0 \mathrm{Gc} / \mathrm{s}$. The results give no evidence for the presence of the water-vapor line. An upper limit on the line intensity, using three standard deviations as a criterion, is 10 percent.

\section{Discussion Following F. D. Drake's Paper}

C. Sagan: What is the maximum permissible depression in the radio spectrum, as allowed by your results?

F. D. Drake: $15^{\circ} \mathrm{K}$.

W. E. Gordon: Are all the data taken at the same time?

F. D. Drake: The eight frequencies observed were observed over and over in a random sequence, with the frequency being changed at about half-hour intervals in the course of a day.

C. Sagan: Do visible terrestrial clouds affect the microwave measurements?

W. J.Welch: We think the answer is yes. Our measurements at or near the water vapor line become affected by sporadic errors when large cumulus clouds are present.

(Paper 69D12-597)

\section{Radiation of Venus at the 13.5-mm Water-Vapor Line}

\section{J. E. Gibson and H. H. Corbett}

E. O. Hulburt Center for Space Research, U.S. Naval Research Laboratory, Washington, D.C.

Brightness temperature measurements of Venus at 13.5-mm wavelength were made following the lower conjunction in November 1962, and also preceding the next closest approach in June 1964. 
The principal objective was to investigate the Cytherean brightness variation between previously observed wavelengths of $8.6 \mathrm{~mm}$ and $3.15 \mathrm{~cm}$, with special interest in the possibility that the 13.5$\mathrm{mm}$ water-vapor absorption maximum might give evidence of water vapor in the atmosphere of Venus. A secondary objective was to investigate the existence of any variation in planetary brightness with phase of solar illumination.

The first group of measurements were on 21 occasions between October 29, 1962, and February 9, 1963, and the second group gave eight measurements between April 26 and June 26, 1964. The observational procedure was much the same as for $8.6 \mathrm{~mm}$ previously [Gibson, 1963]. ${ }^{1}$ The NRL 10-ft reflector was used, and repeated drift scans on each occasion were averaged to obtain measurable deflections. The extinction of the terrestrial atmosphere was determined from readings of sky brightness at a series of elevation angles. The calibration for each occasion was referred to an Argon noise tube, which was compared with a thermal source in the course of separate system tests.

The results indicated that at $13.5-\mathrm{mm}$ wavelength the average disk brightness temperature of Venus is essentially constant at 430 to $440{ }^{\circ} \mathrm{K}$ over a phase variation of 45 to $50 \mathrm{deg}$ each side of lower conjunction, but at greater phase angles up to the limit of measurement just beyond dichotomy the brightness rapidly increases approximately linearly with the fraction of the disk illuminated. No shift in phase between the radio brightness and the solar illumination could be recognized. The results may be stated as follows, where $k$ is the fraction of the disk illuminated, and $i$ is the Sun-Venus-Earth angle:

$$
\begin{aligned}
& T_{b}=430-440^{\circ} \mathrm{K} \text {, for } k<0.19, \text { and } \\
& T_{b}=320^{\circ}+k 600^{\circ} \mathrm{K} \text {, for } 0.6>k>0.19\left(i<130^{\circ}\right) .
\end{aligned}
$$

The variation with phase is such that the brightness temperature at dichotomy $(k=0.5)$ is very close to the mean temperature of $621^{\circ} \mathrm{K}$ at $3.15 \mathrm{~cm}$ found by Mayer, McCullough, and Sloanaker [1962]. ${ }^{2} \quad$ Since measurements at $13.5 \mathrm{~mm}$ could not be continued to near $k=1.0$, it is only possible to extrapolate the measurements obtained, and in the simplest case this gives an average disk temperature of $920^{\circ} \mathrm{K}$. However, this may be modified by the effect of limb darkening, because the negligible variation in brightness temperature for values of $k<0.19$ indicates a temperature variation over the sunlit hemisphere that diminishes to near the dark-side temperature at the terminator. This could have the effect of limiting the maximum average disk brightness temperature to little more than the value reached for $k=0.81$, or $800^{\circ} \mathrm{K}$. In this case the central brightness of the sunlit disk must be greater in order to compensate for the limb darkening.

The brightness temperature of about $435^{\circ} \mathrm{K}$ for the dark hemisphere is comparable to the $410{ }^{\circ} \mathrm{K}$ measured at $8.6 \mathrm{~mm}$, thereby failing to show the decreased temperature at $13.5 \mathrm{~mm}$ that would indicate water vapor absorption of thermal radiation from near the planetary surface. However, comparison of measurements of terrestrial atmospheric attenuation at these two wavelengths shows that the water vapor component is only slightly larger at $13.5 \mathrm{~mm}$, in conflict with theory suggesting a two- to three-fold difference. If the discrepancy is partly attributable to higher absorption than expected at $8.6 \mathrm{~mm}$, the $410{ }^{\circ} \mathrm{K}$ brightness temperature of Venus at this $8.6-\mathrm{mm}$ wavelength is consistent with $350{ }^{\circ} \mathrm{K}$ at $4.3 \mathrm{~mm}$ and $300^{\circ} \mathrm{K}$ at $3.2 \mathrm{~mm}$, on the assumption of atmospheric water vapor and clouds on Venus.

It is expected that a more detailed account of this work will be given elsewhere.

Note added in proof-Recent study of terrestrial atmospheric attenuation data suggests that the unexpectedly high 8.6-mm terrestrial attenuation mentioned above may be due to oxygen rather than water vapor, in which case these terrestrial

${ }^{1}$ Gibson, J. E (1963), Brightness temperature of Venus at $8.6 \mathrm{~mm}$, Astrophys. J. 13 7, 611.

${ }^{2}$ Mayer, C. H., T. P. McCullough, and R. M. Sloanaker (1962), $3.15 \mathrm{~cm}$ observations of Venus in 1961, Mem. Soc. Roy. Sci. Liege 7, 357. 
atmosphere results for water vapor are in reasonable accord with theory. Therefore, as will be shown elsewhere, if the expected frequency-squared dependence of water cloud attenuation is realistic it does not now seem possible to account for the observed 3- to 13.5-mm spectrum of Venus by the single assumption of attenuation in water vapor and water clouds above a high-temperature planetary surface. Since water is believed to have been identified otherwise by several observers $^{3,4}$, the millimeter-wavelength results argue that an additional attenuating component is important in at least the 8- to 9-mm range, or the concept of a thermal origin of microwave radiation near the planetary surface is unsound. The possibility of augmented attenuation in the 8- to 9-mm range is indicated also by the low amplitude of the brightness variation with phase in this wavelength range as reported by other observers, although none of these observations of variation with phase in the 8-mm range appears both reliable and sufficiently extensive. The outstanding remaining question is the explanation of the large brightness variation with phase at $13.5 \mathrm{~mm}$.

(Paper 69D12-598)

${ }^{3}$ Dollfus, A. (1963), Observation of water vapor on the planet Venus. Symposium on origin and evolution of atmospheres and oceans, NASA Institute of Space Studies, New York, 8-9 April.

${ }^{4}$ Bottema, M., W. Plummer, J. Strong, and R. Zander (1965), The composition of the Venus clouds and implications for model atmospheres, J. Geophys. Res. 70, 4401 . 